\title{
Prognostic significance of circulating tumor microemboli in patients with pancreatic ductal adenocarcinoma
}

\author{
GUANGDONG WU, RONGRONG ZHU, YATONG LI, YUPEI ZHAO and MENGHUA DAI \\ Department of General Surgery, Peking Union Medical College Hospital, Beijing 100730, P.R. China
}

Received October 17, 2017; Accepted February 28, 2018

DOI: $10.3892 / \mathrm{ol} .2018 .8264$

\begin{abstract}
The significance of circulating tumor microemboli (CTMs) in patients with pancreatic ductal adenocarcinoma (PDAC) is still unknown. Thus, the present study used a epithelial cellular adhesion molecule independent subtraction and immunostaining-fluorescence in situ hybridization (SET-iFISH) platform to enumerate circulating tumor cells (CTCs) and CTMs in a total of 86 peripheral blood samples from 19 patients with PDAC. The associations between CTCs and CTMs with clinicopathologic factors and prognoses were analyzed. Prior to treatment, CTCs were detected in all 19 patients, and CTMs were detected in 4 patients with different tumor-node-metastasis (TNM) stages. A total of 85 of the 86 peripheral blood samples had cytokeratin 18-negative CTCs. The number of CTCs and CTMs were significantly associated with tumor size and vascular invasion. Patients with CTMs had poorer overall survival and disease-free survival when compared with those without CTMs (7.3 vs. 25.40 months, $\mathrm{P}=0.001$; and 1.80 vs. 18.97 months, $\mathrm{P}=0.037$ ). The presence of CTMs in the peripheral blood prior to surgery was predictive of poor prognosis in PDAC patients. CTMs could be detected in patients of different TNM stage (II, III and IV). Surgery did not benefit patients with CTMs, thus, surgeons should take greater consideration when assessing the requirements for surgery in patients with CTMs.
\end{abstract}

Correspondence to: Dr Menghua Dai, Department of General Surgery, Peking Union Medical College Hospital, 1 Shuaifuyuan Wangfujing, Dongcheng, Beijing 100730, P.R. China

E-mail: daim66@126.com

Abbreviations: CTM, circulating tumor microemboli; PDAC, pancreatic ductal adenocarcinoma; CTCs, circulating tumor cells; SCTCs, small CTCs; EpCAM, epithelial cellular adhesion molecule; CK, cytokeratin; EMT, epithelial-mesenchymal transition; SET-iFISH, EpCAM independent subtraction and immunostaining-fluorescence in situ hybridization; WBCs, white blood cells; CEP8, centromere probe 8; OS, overall survival; ACD, acid citrate dextrose; DFS, disease-free survival

Key words: circulating tumor microemboli, circulating tumor cells, epithelial-mesenchymal transition, pancreatic ductal adenocarcinoma, prognosis

\section{Introduction}

Pancreatic ductal adenocarcinoma (PDAC) is an aggressive and lethal disease with an overall 5-year survival rate of $<5 \%$ (1). In China, more than 90,100 new PDAC cases and an estimated 79,400 deaths from PDAC occurred in 2015 (2). While surgical resection is the most effective treatment for PDAC, most patients have little opportunity for surgery and a poor prognosis due to its aggressive invasion and metastasis. Even if patients have the opportunity for surgery, some are likely to undergo an R1 resection. The majority of patients will develop local or distant tumor recurrence after undergoing a resection. Early detection and dynamic monitoring of the disease progression are difficult. Conventional imaging examinations and carbohydrate antigen 19-9 (CA19-9) assessment often fail to detect the early stage of a primary tumor or metastasis (3). Novel biomarkers are urgently needed for an early diagnosis and detection of disease progression.

Circulating tumor cells (CTCs) are defined as neoplastic cells that are disseminated from primary tumors and secondary deposits, and the detection of CTCs may be a new method to diagnose pancreatic cancer, a so called 'fluid biopsy.' Various CTC isolation technologies have been developed, based on both physical and biological properties. Technology has allowed the identification CTCs using an epithelial marker, such as epithelial cellular adhesion molecule (EpCAM) or cytokeratin (CK), but this method exhibited a very low rate of detection in pancreatic cancer patients $(4,5)$, possibly due to the epithelial-mesenchymal transition (EMT). The EMT has been described as the process in which cancer cells lose some of their epithelial characteristics and gain features of a more mesenchymal phenotype during metastatic progression. This process makes cancer cells more mobile and invasive, which are the main characteristics of CTCs. Epithelial-marker-based technologies potentially miss CTCs with a mesenchymal phenotype, which is important for metastasis. Some studies have employed a size-based or mesenchymal marker to isolate CTCs, which improves the detection rate (6-9).

In this study, we applied a detection platform called EpCAM independent subtraction and immunostaining-fluorescence in situ hybridization (SET-iFISH), which facilitates the improvement of pancreatic CTC detection (10). This platform depletes white blood cells (WBCs) using an anti-CD45 antibody and then identifies CTCs by karyotypic identification of centromere probe 8 (CEP8), which can be performed 
regardless of EpCAM expression and size variations (11). The aim of this study is to investigate whether CTCs and circulating tumor microemboli (CTMs) are related to the clinicopathological factors and overall survival (OS) of PDAC patients.

\section{Materials and methods}

Patients and sample collection. This prospective study was performed at the Peking Union Medical College Hospital (Beijing, China) from August 2014 to April 2015. All research subjects were hospitalized patients or healthy individuals undergoing routine physical examinations. Nineteen PDAC patients (including 3 stage IIa, 11 stage IIb, 4 stage III and 1 stage IV patients) were entered into a prospectively collected database. PDAC was confirmed by pathological assessment. Patients' demographic characteristics, operative parameters, and postoperative outcomes were prospectively collected in the database. Peripheral venous blood $(7.5 \mathrm{ml})$ was collected from each patient in customized acid citrate dextrose (ACD)-anticoagulant tubes (Cytelligen, San Diego, CA, USA) before the operation and at 10 days, 1,3 and 7 months after the operation. All patients were treated with chemotherapy. The blood was processed immediately after collection. This study was approved by the Institutional Review Board of Peking Union Medical College Hospital and written informed consent was obtained from all subjects.

Enrichment and identification of CTCs. The process of the enrichment and identification of pancreatic CTCs was performed according to the kit instructions (Cytelligen). In brief, $7.5 \mathrm{ml}$ of peripheral venous blood was centrifuged to deplete the serum. The remaining components were mixed with hCTCs Separation Matrix and centrifuged to remove red blood cells. The remaining sample was incubated with immunomagnetic particles conjugated to anti-CD45 monoclonal antibodies (Cytelligen) for the separation of WBCs. The resulting cell pellet was placed onto CTCs coated slides for iFISH. Hybridization solution containing a CEP8 probe labeled with Spectrum Orange (Cytelligen) was added to the slides, which were mounted and denatured. Then, the cells were incubated with monoclonal anti-CK18 conjugated to Alexa Fluor 488 and monoclonal anti-CD45 conjugated to Alexa Fluor 594 (Cytelligen). Finally, the nuclear dye 4',6-diamidino-2-phenylindole (Cytelligen) was added, and the slides were subsequently subjected to microscopic observation.

Statistical analysis. Continuous variables are expressed as the mean \pm standard deviation or the median (minimum, maximum), and categorical variables are expressed as percentages. Continuous variables were compared using the independent-samples $\mathrm{T}$ test and Wilcoxon rank test, and categorical variables were compared using the Pearson chi-square test. The primary end point was OS, which was calculated using the Kaplan-Meier method. Survival differences were assessed using the log-rank test. Two-sided $\mathrm{P}<0.05$ was considered to indicate a statistically significant difference. All statistical analyses were conducted by using IBM SPSS version 18.0 (BM Corp., Armonk, NY, USA).

\section{Results}

Identification of CTCs patterns in PDAC patients. Cells from the peripheral blood samples of PDAC patients were identified using the SET-iFISH platform. Based on the immunostaining of an epithelial marker (CK18), a WBC marker (CD45), the cell nucleus (DAPI) and different numbers of chromosome ploidy (CEP 8), CTCs were defined with the following three patterns: A, CK18+/CD45-/DAPI+/CEP8 $\neq 2$; B, CK18-/CD45-/DAPI+/ $\mathrm{CEP} 8 \neq 2$; and C, CK18+/CD45-/DAPI+/CEP8=2 . All CD45+ cells were defined as WBCs, and CK-/CD45-/DAPI+/CEP8=2 cells were defined as indeterminate cells $(11,12)$. We detected these markers in 86 peripheral blood samples from 19 PDAC patients at different time points. Several peripheral blood samples were not detected because some patients were not able to meet our request on time due to their personal reasons or deaths. Of these 86 samples, only one sample was detected as having CK18+ CTCs preoperatively, while the others were all negative for these cells. Furthermore, we found some CTCs that were similar in size to WBCs, which we called small CTCs (SCTCs), and we isolated some clusters of CTCs called CTMs (Fig. 1). We did not detect CK18+ and CD45+ in all the CTMs.

CTCs in PDAC patients. All 19 PDAC patients included in this study were histologically confirmed as having PDAC. The patient cohort consisted of $47.4 \%$ males, with an average age of 59.1 years (range, 40-78 years). Preoperative CA19-9 levels were measured in all patients. The mean CA19-9 level was 321.6 units/ml (range: 0.6-2074 units/ml), and 14 patients (73.7\%) had a level greater than 37 units $/ \mathrm{ml}$, which is considered abnormally elevated. Sixteen $(84.2 \%)$ patients underwent resection, and $3(15.8 \%)$ patients had unresectable disease. Of the patients who underwent operation, $62.5 \%$ underwent resection by pancreaticoduodenectomy $(n=10)$ and $6(37.5 \%)$ had a distal pancreatectomy with splenectomy. The average tumor size was $3.39 \mathrm{~cm}$ (range, $1.2-7 \mathrm{~cm}$ ). The majority of the adenocarcinomas were either moderately or poorly differentiated $(n=11,68.7 \%)$. Thirteen $(81.3 \%)$ patients had positive regional lymph nodes. After analyzing the numbers of CTCs with the above clinicopathologic features, no statistically significant differences were found in regard to the age and sex of the patients and the stage, grade and status of the lymph nodes. The number of CTCs was significantly greater in patients with a tumor size $>3 \mathrm{~cm}$, perineural invasion or vascular invasion. No significant differences were found in the numbers of SCTCs regarding any of the clinicopathologic features (Table I).

CTMs in PDAC patients. CTMs were found in five patients (three stage IIb patients, one stage III patient and one stage IV patient). Four of these five patients were found CTMs before treatment. Before treatment, a concentration of one CTM per $7.5 \mathrm{ml}$ of blood was found in two patients (one stage III patient and one stage IV patient), and concentrations of three CTMs and six CTMs per $7.5 \mathrm{ml}$ of blood were found in two stage IIb patients, which showed that the number of CTMS was not significantly different with TNM stage. No statistically significant differences were observed in the numbers of CTMs in regard to the age and sex of the patients and the stage, grade and status of the lymph nodes. The number of CTMs was 

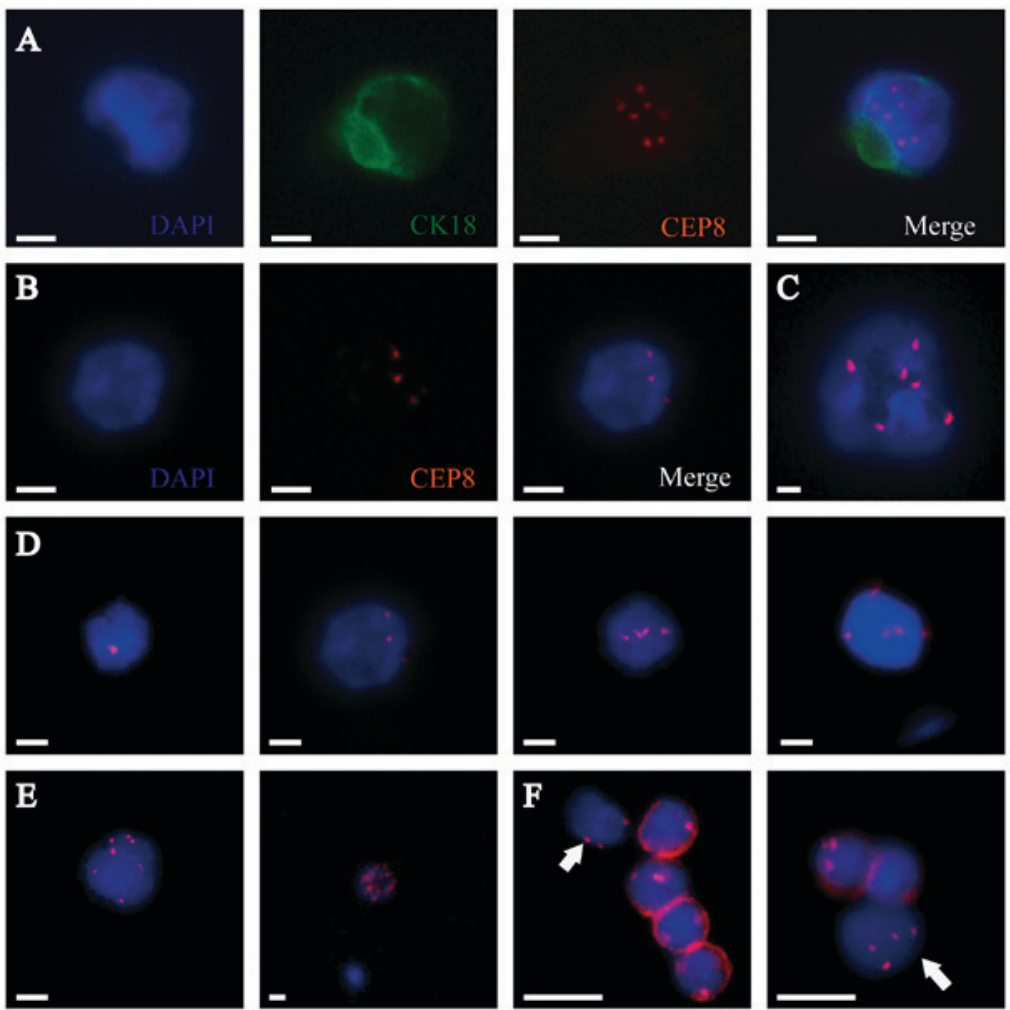
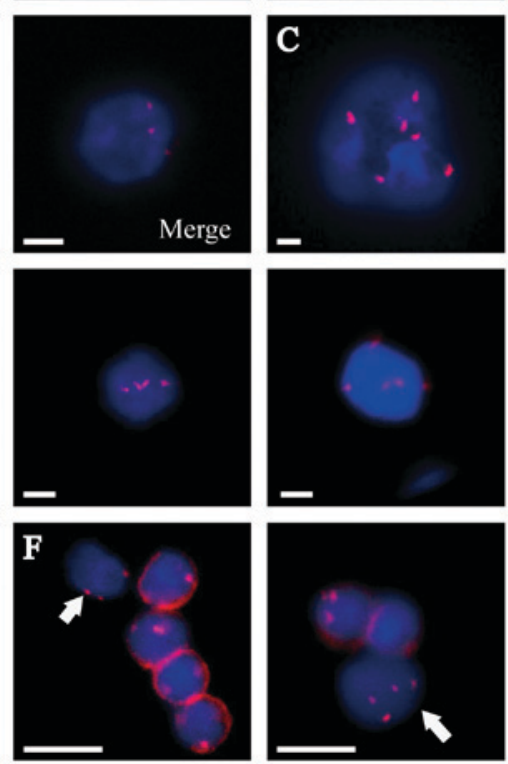

Figure 1. Identification of CTCs in PDAC samples by the SET-iFISH platform. CK18, green; CEP8, orange; DAPI, blue; and CD45, red. (A) CTC: CK18+/CD45-/DAPI+/CEP8=6. (B) CTC: CK18-/CD45-/DAPI+/CEP8=3. (C) CTM: Clusters of CTCs. (D) CTCs with different numbers of CEP8 (CEP8=1, 3,4 and 5, respectively). (E) CTCs with CEP8 $>5$. (F) Small CTC: The diameter of the CTC is $<5 \mu \mathrm{m}$, which is similar to a white blood cell (indicated by white arrows). Scale bars $=5 \mu \mathrm{m}$. CTCs, circulating tumor cells; PDAC, pancreatic ductal adenocarcinoma; CK, cytokeratin; CEP8, centromere probe 8; CD, cluster of differentiation.

significantly greater in patients with a tumor size $>3 \mathrm{~cm}$ and vascular invasion (Table I).

Dynamic changes in CTCs and CTMs in PDAC patients. This study detected CTCs in 86 peripheral blood samples collected at different times from 19 PDAC patients. Of all peripheral blood samples, 19 samples were obtained preoperatively, 19 samples were obtained at 10 days postoperation, 18 samples were obtained at 1 month postoperation, 15 samples were obtained at 3 months postoperation ( 2 cycles of adjuvant gemcitabine therapy) and 15 samples were obtained at 7 months postoperation (6 cycles of adjuvant gemcitabine therapy). We found that the number of CTCs after surgery or chemotherapy was not significantly different than the number of preoperative CTCs. Only samples obtained at 10 days postoperation showed significantly different numbers of SCTCs than samples obtained preoperatively (Table II).

In four of five patients, CTMs were detected before treatment. One month after surgery, the number of CTMs in a stage III patient increased to 6 per $7.5 \mathrm{ml}$ of blood, and one CTM was detected in a new patient (stage IIb) who was negative before surgery. After 2 cycles of adjuvant gemcitabine therapy, CTMs were detected in two patients who were dead at approximately 7 months postoperation, while after 6 cycles of adjuvant gemcitabine therapy, two CTMs were found in one patient who had 3 CTMs detected per $7.5 \mathrm{ml}$ of blood before surgery (Table III).

Survival analysis. The mean and median follow-up times of all patients were 19.94 and 24.90 months, respectively. During the follow-up period, 13 patients died due to disease progression, including 3 patients who did not undergo surgery. two patients remained alive but exhibited disease progression. Four patients remained alive without evidence of disease progression. No patients were lost to follow-up. To evaluate the influence of the cutoff value of the CTCs count on the hazard ratios of OS, different values for the number of CTCs per $7.5 \mathrm{ml}$ were tested for correlation with OS using the Kaplan-Meier method. However, the number of CTCs was not associated with survival. We also examined the correlation between OS and the number of SCTCs, but no prognostic significance was found. The relationship between the number of CTMs and survival was further explored. Patients with no CTMs survived significantly longer than those with CTMs (25.4 vs. 7.3 months, $\mathrm{P}=0.001)$ (Table IV) (Fig. 2). The median disease-free survival (DFS) of the patients with CTMs was significantly shorter than that of patients without CTMs (18.97 vs. 1.8 months, $\mathrm{P}=0.037$ ) (Fig. 3). The effects of tumor size, grade of differentiation, TNM stage, perineural and vascular invasion, resection margin status, and adjuvant chemoradiation therapy on median OS were all evaluated using the Kaplan-Meier method. Survival differences were assessed using the log-rank test (Table III). The results of these analyses indicated that TNM stage and vascular invasion were significantly associated with OS.

\section{Discussion}

PDAC has a dismal prognosis, which is commonly due to the occurrence of invasion and metastasis before this 
Table I. Patient characteristics and numbers of CTCs, CTMs and SCTCs.

\begin{tabular}{|c|c|c|c|c|c|c|c|}
\hline Variable & $\begin{array}{l}\text { All patients } \\
\qquad(\mathrm{n}=19)\end{array}$ & $\begin{array}{c}\text { CTCs, n } \\
(\min , \max )\end{array}$ & P-value & $\begin{array}{c}\text { CTMs, n } \\
(\min , \max )\end{array}$ & P-value & $\begin{array}{l}\text { SCTCs, n } \\
(\min , \max )\end{array}$ & P-value \\
\hline Age (years) & & & 0.383 & & 0.908 & & 0.818 \\
\hline$\leq 60$ & $11(57.9 \%)$ & $6(1,30)$ & & $0(0,6)$ & & $1(1,3)$ & \\
\hline$>60$ & $8(42.1 \%)$ & $4(1,22)$ & & $0(0,1)$ & & $1(0,10)$ & \\
\hline Gender & & & 0.511 & & 0.909 & & 0.316 \\
\hline Male & $9(47.4 \%)$ & $5(1,22)$ & & $0(0,1)$ & & $1(0,10)$ & \\
\hline Female & $10(52.6 \%)$ & $7(1,30)$ & & $0(0,6)$ & & $1(0,3)$ & \\
\hline Tumor size $(\mathrm{cm}),(\mathrm{n}=16)^{\mathrm{a}}$ & & & $0.019^{\mathrm{b}}$ & & $0.036^{\mathrm{b}}$ & & 0.855 \\
\hline$\leq 3$ & $9(56.3 \%)$ & $3(1,9)$ & & $0(0,0)$ & & $1(0,3)$ & \\
\hline$>3$ & $7(43.7 \%)$ & $17(1,30)$ & & $0(0,6)$ & & $1(1,10)$ & \\
\hline CA19-9 level (units/ml) & & & 0.963 & & 1.000 & & 0.570 \\
\hline$<37$ & $5(26.3 \%)$ & $6(2,17)$ & & $0(0,3)$ & & $1(1,3)$ & \\
\hline$>37$ & $14(73.7 \%)$ & $5(1,30)$ & & $0(0,6)$ & & $1(0,10)$ & \\
\hline TNM Stage & & & 0.852 & & 0.363 & & 0.717 \\
\hline I-II & $14(73.7 \%)$ & $5.5(1,30)$ & & $0(0,6)$ & & $1(0,3)$ & \\
\hline III-IV & $5(26.3 \%)$ & $5(1,22)$ & & $0(0,1)$ & & $1(0,10)$ & \\
\hline Differentiation $(n=16)$ & & & 0.078 & & 0.213 & & 0.192 \\
\hline Well/moderate & $11(68.7 \%)$ & $6(1,30)$ & & $0(0,6)$ & & $1(0,10)$ & \\
\hline Poor & $5(31.3 \%)$ & $2(1,5)$ & & $0(0,0)$ & & $1(1,1)$ & \\
\hline Perineural invasion $(n=16)$ & & & $0.017^{\mathrm{b}}$ & & 0.934 & & 0.078 \\
\hline No & $11(68.7 \%)$ & $3(1,21)$ & & $0(0,6)$ & & $1(0,3)$ & \\
\hline Yes & $5(31.3 \%)$ & $15(6,30)$ & & $0(0,1)$ & & $2(1,10)$ & \\
\hline Vascular invasion $(n=16)$ & & & $0.043^{\mathrm{b}}$ & & $0.036^{\mathrm{b}}$ & & 0.583 \\
\hline No & $9(56.3 \%)$ & $3(1,9)$ & & $0(0,0)$ & & $1(0,3)$ & \\
\hline Yes & $7(43.7 \%)$ & $17(1,30)$ & & $0(0,6)$ & & $1(1,10)$ & \\
\hline Positive lymph nodes $(n=16)$ & & & 0.199 & & 0.374 & & 0.215 \\
\hline No & $3(18.7 \%)$ & $6(6,30)$ & & $0(0,0)$ & & $2(1,3)$ & \\
\hline Yes & $13(81.3 \%)$ & $3(1,22)$ & & $0(0,6)$ & & $1(0,10)$ & \\
\hline
\end{tabular}

${ }^{\mathrm{a} D i v i d e d}$ into 2 groups, according to the median. ${ }^{\mathrm{b}} \mathrm{P}<0.05$. CTCs, circulating tumor cells; SCTC, small CTCs; CTMs, circulating tumor microemboli; TNM, tumor-node-metastasis.

Table II. Number of CTCs, CTM and SCTCs at different times.

\begin{tabular}{|c|c|c|c|c|c|c|c|}
\hline Time & $\begin{array}{c}\text { Number of } \\
\text { samples }\end{array}$ & $\begin{array}{c}\text { CTCs, n } \\
(\min , \max )\end{array}$ & Difference & $\begin{array}{c}\text { CTM, n } \\
(\min , \max )\end{array}$ & Difference & $\begin{array}{l}\text { SCTCs, n } \\
(\min , \max )\end{array}$ & Difference \\
\hline Pre-operative & 19 & $5(1,30)$ & & $0(0,6)$ & & $1(0,10)$ & \\
\hline 10 days postoperation & 19 & $13(6,19)$ & 0.098 & $0(0,1)$ & 0.484 & $4(0,11)$ & $0.013^{\mathrm{a}}$ \\
\hline 1 month postoperation & 18 & $2.5(0,30)$ & 0.267 & $0(0,6)$ & 0.974 & $1(0,6)$ & 0.365 \\
\hline 3 months postoperation & 15 & $0(0,24)$ & 0.353 & $0(0,0)$ & 0.273 & $0(0,21)$ & 0.573 \\
\hline 7 months postoperation & 15 & $5.5(2,10)$ & 0.935 & $0(0,2)$ & 0.866 & $2.5(0,7)$ & 0.321 \\
\hline
\end{tabular}

${ }^{\mathrm{a}} \mathrm{P}<0.05$. CTM, circulating tumor microemboli; CTCs, circulating tumor cells; SCTCs.

disease is diagnosed. In recent years, CTCs have attracted increasing attention as a novel biomarker in clinical practice due to their use in the early detection of tumors. Some studies have shown that CTCs are present in pancreatic cancer (12-14). A study on pancreatic cancer revealed that CTCs can enter the bloodstream before tumor formation occurs (15). However, it is noteworthy that in the past, most studies or clinical applications have employed the CellSearch 
Table III. CTM in 5 patients with pancreatic ductal adenocarcinoma.

\begin{tabular}{llccccccc}
\hline Patient & $\begin{array}{c}\text { Recurrence } \\
\text { site }\end{array}$ & $\begin{array}{c}\text { DFS } \\
\text { (months) }\end{array}$ & $\begin{array}{c}\text { OS } \\
\text { (months) }\end{array}$ & $\begin{array}{c}\text { Prior to } \\
\text { treatment }\end{array}$ & $\begin{array}{c}10 \text { days } \\
\text { po }\end{array}$ & $\begin{array}{c}1 \text { months } \\
\text { po }\end{array}$ & $\begin{array}{c}3 \text { months } \\
\text { po }\end{array}$ & $\begin{array}{c}7 \text { months } \\
\text { po }\end{array}$ \\
\hline Patient 1 (stage IIb) & Liver & 1.8 & 7.3 & 6 CTMs & 1 CTMs & 0 CTMs & 1 CTMs & $/$ \\
Patient 2 (stage IIb) & Liver & 3.2 & 20.8 & 3 CTMs & 0 CTMs & 0 CTMs & 0 CTMs & 2 CTMs \\
Patient 3 (stage III) & Liver and lung & 1.8 & 3.9 & 1 CTMs & 0 CTMs & 6 CTMs & $/$ & $/$ \\
Patient 4 (stage IV) & - & - & 7.2 & 1 CTMs & 0 CTMs & 0 CTMs & 3 CTMs & $/$ \\
Patient 5 (stage IIb) & Liver & 4.2 & 10.6 & 0 CTMs & 0 CTMs & 1 CTMs & 0 CTMs & 0 CTMs \\
\hline
\end{tabular}

Patient 4 did not receive surgery however, did undergo chemotherapy due to liver metastasis; therefore, there is no recurrence data for this patient. DFS, disease-free survival; OS, overall survival; po, postoperation; CTMs, circulating tumor microemboli; /, peripheral venous blood was not collected from the patient due to mortality.

Table IV. Kaplan-Meier analysis of OS.

\begin{tabular}{llc}
\hline & \multicolumn{2}{c}{ Univariate analysis } \\
\cline { 2 - 3 } Factor & $\begin{array}{c}\text { Median OS } \\
\text { (months) }\end{array}$ & P-value \\
\hline CA19-9 level $(<37 />37)(\mathrm{U} / \mathrm{ml})$ & $-/ 14.47$ & 0.100 \\
Tumor size $(\leq 3 \mathrm{~cm} />3 \mathrm{~cm})^{\mathrm{a}}$ & $10.60 / 28.97$ & 0.187 \\
Differentiation (well-moderate/poor) & $24.90 / 25.40$ & 0.812 \\
TNM (I-II/III-IV) & $25.40 / 9.50$ & $0.008^{\mathrm{c}}$ \\
Perineural invasion (no/yes) & $28.97 / 25.03$ & 0.800 \\
Vascular invasion (no/yes) & $28.97 / 10.60$ & $0.017^{\mathrm{b}}$ \\
Resection margin status (R0/not R0) & $25.40 / 7.30$ & 0.194 \\
CTMs (no/yes) & $25.40 / 7.30$ & $0.001^{\mathrm{c}}$ \\
\hline
\end{tabular}

${ }^{\mathrm{a} D i v i d e d}$ into 2 groups, according to the median; ${ }^{\mathrm{b}} \mathrm{P}<0.05$ and ${ }^{\mathrm{c}} \mathrm{P}<0.01$. CA19-9, cancer antigen 19-9; TNM, tumor-node-netastasis; CTMs, circulating tumor microemboli; OS, overall survival.

system, which depends upon the principle that CTCs can be captured via an anti-EpCAM antibody. This method of CTCs capture is based on epithelial expression and may result in decreased sensitivity and loss of key information due to the EMT process, which has is considered a critical factor in tumor cell invasion and metastasis (16). The detection of epithelial-marker-negative CTCs is very important for patients. A study by Poruk et al (14) assessed CTCs based on epithelial and mesenchymal markers in PDAC patients and found that heterogeneous CTCs provide prognostic utility for PDAC patients. Therefore, in our study, we detected CTCs using the SET-iFISH platform instead of epithelial markers. Surprisingly, we found that only one of 46 samples from the 19 patients exhibited epithelial CTCs along with CTMs in the peripheral blood. If the traditional CellSearch system had been used, this important information would have been missed.

In this study, the relationships of CTCs and CTMs with clinicopathologic data were investigated. The numbers of CTCs and CTMs were significantly associated with tumor size and vascular invasion. CTCs, but not CTMs, were

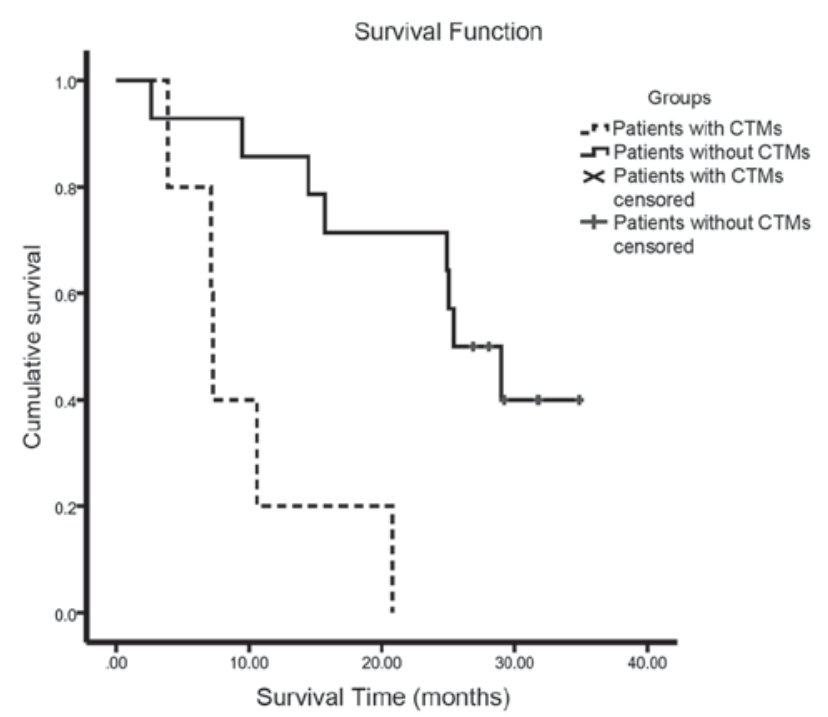

Figure 2. Comparison of overall survival function between patients with CTMs and patients without CTMs by Kaplan-Meier analysis. CTM, circulating tumor microemboli.

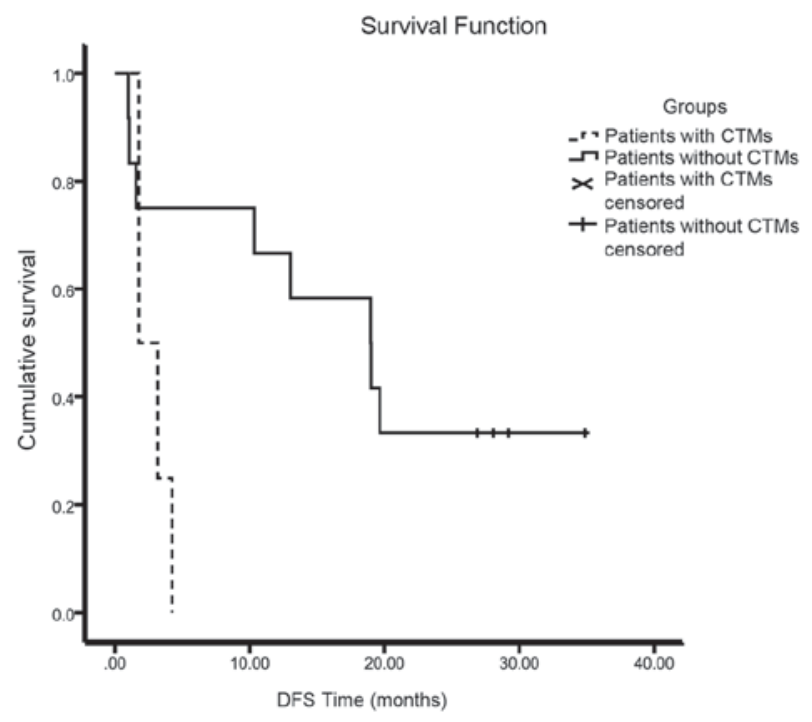

Figure 3. Comparison of DFS function between patients with CTMs and patients without CTMs by Kaplan-Meier analysis. DFS, disease-free survival; CTM, circulating tumor microemboli. 
associated with perineural invasion. Although the reasons for these relationships are unknown, these findings could reflect the severity of the illness and the effect that tumors have on vascular or perineural invasion. Zhang et al (11) also found no correlations between CTCs and the TNM stage, CA19-9 level or lymph node status using the same platform. The results of our study were consistent with this finding by Zhang et al (11). The lack of correlation between CTCs and TNM stage was also demonstrated by the presence of CTCs in patients with different stages of PDAC $(17,18)$. CA19-9 is the most commonly used tumor marker of pancreatic cancer, but it was not elevated in a Lewis ${ }^{\mathrm{a}-\mathrm{b}-}$ patient. CTCs and CTMs were detected in patients with a normal CA19-9 level in our study, which suggested that CTC and CTMs detection combined with CA19-9 levels could improve the diagnosis rate $(12,13)$. The number of CTCs, especially the number of SCTCs, was increased 10 days after the operation compared with the level before surgery. Another study also found the same phenomenon using SET-iFISH (11). Some studies employing epithelial markers to detect CTCs also showed a similar finding $(19,20)$. They proposed that the change in the number of CTCs might be associated with dormant disseminated tumor cells being reactivated and released into the circulation after the primary tumors were removed. The reason for this phenomenon, which could involve the stress of surgery, is still unknown.

Although several follow-up studies have confirmed the general prognostic value of CTCs, the prognostic significance of CTCs in pancreatic cancer is still controversial. A diversified detection platform obtained different conclusions, and even results obtained using the same detection platform were inconsistent. CTCs positive patients were shown to have a shorter progression-free survival in one study. However, another study arrived at the opposite conclusion using the same CellSearch system $(4,5)$. The size-based CTCs detection platform also yielded different conclusions. Recently, a study using the Isolation by Size of Epithelial Tumor cells method found that CTCs could provide prognostic utility for PDAC patients (14). Another study adopting a similar size-based device failed to reveal the prognostic significance of CTCs (21). Some studies using the SET-iFISH platform showed that CTCs positive pancreatic cancer patients exhibit a worse survival rate $(11,12)$; however, in this study, we did not find this correlation. Further studies are needed to analyze this relationship.

Despite that CTCs were not associated with the prognosis in our study, the presence of CTMs in patients was significantly associated with a worse prognosis, which was consistent with the results of a previous study (22). The authors found that the number of CTMs, instead of CTCs, before treatment was an independent predictor of OS in PDAC patients. In another study, the presence of CTMs was used as an independent prognostic marker in small cell lung cancer patients and was correlated with a worse clinical outcome (23). Why do the patients with CTMs have a dismal prognosis? Using mouse models with tagged mammary tumors, Aceto et al (24) demonstrated that CTCs clusters are oligoclonal precursors. Although they are rare in the circulation compared with single CTCs, CTC clusters have a 23- to 50-fold increased metastatic potential (24). Some studies also speculate that tumor cells form CTMs to resist anoikis, which is a survival advantage $(25,26)$. The increased metastatic potential and resistance of anoikis might explain why patients with CTMs had a worse prognosis in the present study.

In this study, the five patients with CTMs had different TNM stages, and four of these patients underwent surgery. The median OS of these patients was only 7.3 months. As the previous studies have described, the median OS of metastatic pancreatic cancer patients who undergo chemotherapy (gemcitabine, nab-Paclitaxel plus Gemcitabine or FOLFIRINOX) is 6.7-11.1 months $(27,28)$, which is equivalent to or even better than that of patients with CTMs in this study. Therefore, we presumed that CTMs may be micrometastasis, which is irrelevant to the clinical TNM stage. The TNM stage is mainly determined according to imaging examinations before surgery at the present time, which provide a reference for surgeons. However, this micrometastasis in the blood cannot be detected by a traditional clinical examination. Patients with CTMs exhibited very short survival after surgery, which suggests that the surgeons should cautiously consider surgery for patients with CTMs.

Compared with CTCs, CTMs effectively reflected the severity of illness and dismal prognosis of patients in our study. Although CTCs have been found in many studies, only a few successful detections of CTC clusters have been reported, especially in PDAC patients (29). We believe that the role of CTMs in current clinical applications and research is largely underestimated. However, this study has a small sample size of only 19 patients, most of whom had either stage II or III disease and the follow-up time is limited. Thus, a further large-scale study is needed. CTMs should be given more attention because their detection could be a powerful tool that can benefit patients.

We used the SET-iFISH platform to detect CTCs and CTMs in various pathological stages of pancreatic cancer. Unlike in other studies, most of the CTCs in our study were CK18 negative. CTMs effectively reflected the severity of the illness and the dismal prognosis of patients and might be a new biomarker of PDAC, independent of the current staging system.

\section{Acknowledgements}

Not applicable.

\section{Funding}

No funding was received.

\section{Availability of data and materials}

All data generated or analyzed during this study are included in this published article.

\section{Authors' contributions}

GW performed the experiments, analyzed the data and wrote the manuscript. RZ contributed towards the acquisition of the data and manuscript preparation. YL contributed to the interpretation of survival data. YZ assisted with the conception of the study and revised the manuscript. MD contributed to the conception of the study, designed the experiments and approved the final version of the manuscript. 


\section{Ethics approval and consent to participate}

The present study was approved by the Institutional Review Board of Peking Union Medical College Hospital (Beijing, China) and written informed consent was obtained from all subjects.

\section{Consent for publication}

Written informed consent was obtained from all patients enrolled.

\section{Competing interests}

The authors declare that they have no competing interests.

\section{References}

1. Siegel RL, Miller KD and Jemal A: Cancer statistics, 2016. CA Cancer J Clin 66: 7-30, 2016.

2. Chen W, Zheng R, Baade PD, Zhang S, Zeng H, Bray F, Jemal A, Yu XQ and He J: Cancer statistics in China, 2015. CA Cancer J Clin 66: 115-132, 2016.

3. Chari ST, Kelly K, Hollingsworth MA, Thayer SP, Ahlquist DA, Andersen DK, Batra SK, Brentnall TA, Canto M, Cleeter DF, et al Early detection of sporadic pancreatic cancer: Summative review. Pancreas 44: 693-712, 2015.

4. Bissolati M, Sandri MT, Burtulo G, Zorzino L, Balzano G and Braga M: Portal vein-circulating tumor cells predict liver metastases in patients with resectable pancreatic cancer. Tumour Biol 36: 991-996, 2015.

5. Bidard FC, Huguet F, Louvet C, Mineur L, Bouché O, Chibaudel B, Artru P, Desseigne F, Bachet JB, Mathiot C, et al: Circulating tumor cells in locally advanced pancreatic adenocarcinoma: The ancillary CirCe 07 study to the LAP 07 trial. Ann Oncol 24: 2057-2061, 2013.

6. Lin HK, Zheng S, Williams AJ, Balic M, Groshen S, Scher HI, Fleisher M, Stadler W, Datar RH, Tai YC and Cote RJ: Portable filter-based microdevice for detection and characterization of circulating tumor cells. Clin Cancer Res 16: 5011-5018, 2010.

7. Harouaka RA, Zhou MD, Yeh YT, Khan WJ, Das A, Liu X, Christ CC, Dicker DT, Baney TS, Kaifi JT, et al: Flexible micro spring array device for high-throughput enrichment of viable circulating tumor cells. Clin Chem 60: 323-333, 2014.

8. Lecharpentier A, Vielh P, Perez-Moreno P, Planchard D, Soria JC and Farace F: Detection of circulating tumour cells with a hybrid (epithelial/mesenchymal) phenotype in patients with metastatic non-small cell lung cancer. Br J Cancer 105: 1338-1341, 2011

9. Armstrong AJ, Marengo MS, Oltean S, Kemeny G, Bitting RL, Turnbull JD, Herold CI, Marcom PK, George DJ and Garcia-Blanco MA: Circulating tumor cells from patients with advanced prostate and breast cancer display both epithelial and mesenchymal markers. Mol Cancer Res 9: 997-1007, 2011.

10. Lin PP: Integrated EpCAM-independent subtraction enrichment and iFISH strategies to detect and classify disseminated and circulating tumors cells. Clin Transl Med 4: 38, 2015.

11. Zhang Y, Wang F, Ning N, Chen Q, Yang Z, Guo Y, Xu D, Zhang D, Zhan T and Cui W: Patterns of circulating tumor cells identified by CEP8, CK and CD45 in pancreatic cancer. Int J Cancer 136: 1228-1233, 2015.

12. Gao Y, Zhu Y, Zhang Z, Zhang C, Huang X and Yuan Z: Clinical significance of pancreatic circulating tumor cells using combined negative enrichment and immunostaining-fluorescence in situ hybridization. J Exp Clin Cancer Res 35: 66, 2016.
13. Kulemann B, Pitman MB, Liss AS, Valsangkar N, Fernández-Del Castillo C, Lillemoe KD, Hoeppner J, Mino-Kenudson M, Warshaw AL and Thayer SP: Circulating tumor cells found in patients with localized and advanced pancreatic cancer. Pancreas 44: 547-550, 2015

14. Poruk KE, Valero V III, Saunders T, Blackford AL, Griffin JF, Poling J, Hruban RH, Anders RA, Herman J, Zheng L, et al: Circulating tumor cell phenotype predicts recurrence and survival in pancreatic adenocarcinoma. Ann Surg 264: 1073-1081, 2016.

15. Rhim AD, Mirek ET, Aiello NM, Maitra A, Bailey JM, McAllister F, Reichert M, Beatty GL, Rustgi AK, Vonderheide RH, et al: EMT and dissemination precede pancreatic tumor formation. Cell 148: 349-361, 2012.

16. Thiery JP: Epithelial-mesenchymal transitions in tumour progression. Nat Rev Cancer 2: 442-454, 2002.

17. Tjensvoll K, Nordgård $\mathrm{O}$ and Smaaland R: Circulating tumor cells in pancreatic cancer patients: Methods of detection and clinical implications. Int J Cancer 134: 1-8, 2014.

18. Nagrath S, Sequist LV, Maheswaran S, Bell DW, Irimia D, Ulkus L, Smith MR, Kwak EL, Digumarthy S, Muzikansky A, et al: Isolation of rare circulating tumour cells in cancer patients by microchip technology. Nature 450: 1235-1239, 2007.

19. Sergeant G, Roskams T, van Pelt J, Houtmeyers F, Aerts R and Topal B: Perioperative cancer cell dissemination detected with a real-time RT-PCR assay for EpCAM is not associated with worse prognosis in pancreatic ductal adenocarcinoma. BMC Cancer 11: 47, 2011.

20. Sandri MT, Zorzino L, Cassatella MC, Bassi F, Luini A, Casadio C, Botteri E, Rotmensz N, Adamoli L and Nolè F: Changes in circulating tumor cell detection in patients with localized breast cancer before and after surgery. Ann Surg Oncol 17: $1539-1545,2010$

21. Kulemann B, Liss AS, Warshaw AL, Seifert S, Bronsert P, Glatz T, Pitman MB and Hoeppner J: KRAS mutations in pancreatic circulating tumor cells: A pilot study. Tumour Biol 37: 7547-7554, 2016.

22. Chang MC, Chang YT, Chen JY, Jeng YM, Yang CY, Tien YW, Yang SH, Chen HL, Liang TY, Wang CF, et al: Clinical significance of circulating tumor microemboli as a prognostic marker in patients with pancreatic ductal adenocarcinoma. Clin Chem 62: 505-513, 2016.

23. Hou JM, Krebs MG, Lancashire L, Sloane R, Backen A, Swain RK, Priest LJ, Greystoke A, Zhou C, Morris K, et al: Clinical significance and molecular characteristics of circulating tumor cells and circulating tumor microemboli in patients with small-cell lung cancer. J Clin Oncol 30: 525-532, 2012.

24. Aceto N, Bardia A, Miyamoto DT, Donaldson MC, Wittner BS, Spencer JA, Yu M, Pely A, Engstrom A, Zhu H, et al: Circulating tumor cell clusters are oligoclonal precursors of breast cancer metastasis. Cell 158: 1110-1122, 2014.

25. Liotta LA, Saidel MG and Kleinerman J: The significance of hematogenous tumor cell clumps in the metastatic process. Cancer Res 36: 889-894, 1976.

26. Friedl $\mathrm{P}$ and Gilmour D: Collective cell migration in morphogenesis, regeneration and cancer. Nat Rev Mol Cell Biol 10: 445-457, 2009.

27. Conroy T, Desseigne F, Ychou M, Bouché O, Guimbaud R, Bécouarn Y, Adenis A, Raoul JL, Gourgou-Bourgade S, de la Fouchardière $\mathrm{C}$, et al: FOLFIRINOX versus gemcitabine for metastatic pancreatic cancer. N Engl J Med 364: 1817-1825, 2011.

28. Von Hoff DD, Ervin T, Arena FP, Chiorean EG, Infante J, Moore M, Seay T, Tjulandin SA, Ma WW, Saleh MN, et al: Increased survival in pancreatic cancer with nab-paclitaxel plus gemcitabine. N Engl J Med 369: 1691-1703, 2013.

29. Hong Y, Li Z and Zhang Q: A circulating tumor cell cluster-based model for tumor metastasis (Hypothesis). Oncology Lett 12: 4891-4895, 2016. 